\title{
Spacecraft Launch Depressurization Loads
}

\author{
Ángel Sanz-Andrés, Julián Santiago-Prowald, Antonio Ayuso-Barea
}

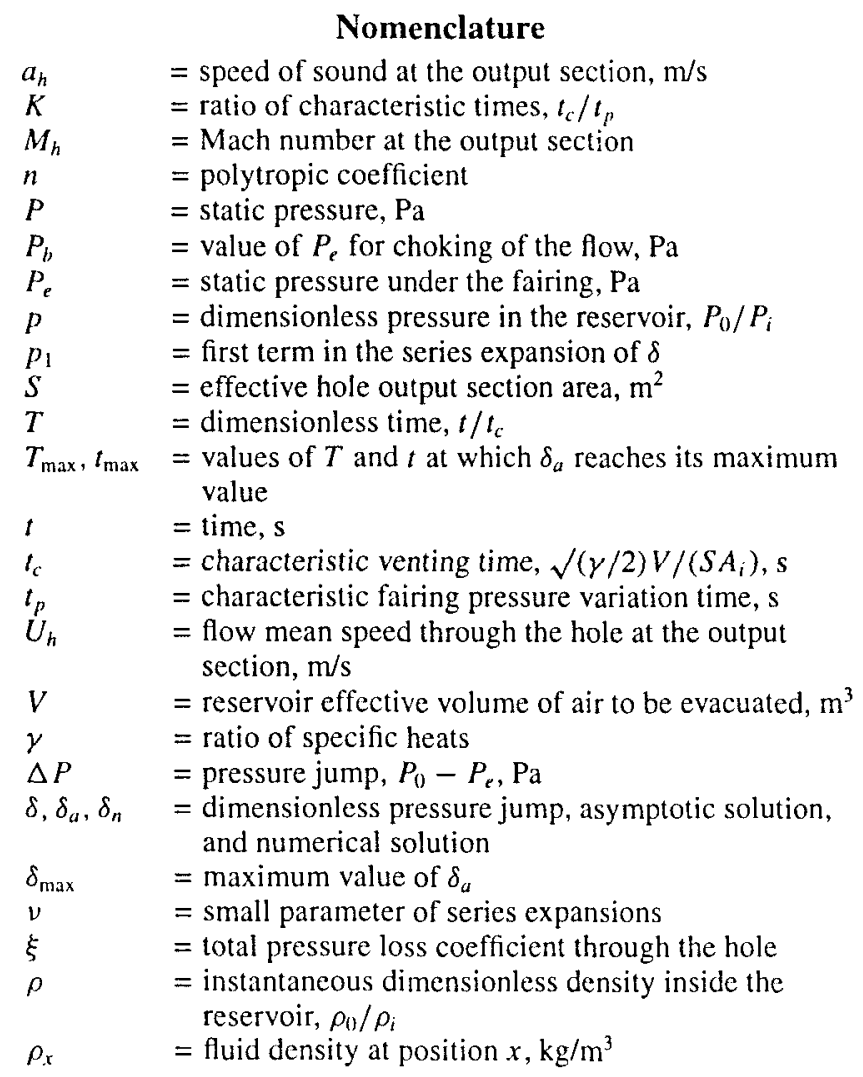

Subscripts

$=$ instantaneous conditions inside the fairing

$=$ instantaneous conditions at the reservoir hole exit

$=$ initial conditions in the reservoir

$=$ instantancous reservoir conditions

\section{Introduction}

$\mathbf{T}$ HE mechanical loads applied on the structural elements of a satellite and its components, usually classified as quasistatic and dynamic loads, depend on accelerations, vibrations, and shocks, but also pressure loads during the launch must be taken into account. ${ }^{.}$These pressure loads are a function of the evolution (in attitude and height) of the launch vehicle through the atmosphere and the venting rates of the fairing and the spacecraft. Because of the presence of the fairing, only the static pressure profiles belong to the environmental data necessary for the design and testing of spacecraft.

Air trapped in the spacecraft or any component is at a higher pressure than the surroundings until the discharge is completed. The venting rate depends on the static pressure difference between spacecraft and fairing and on the size, shape, number, and distribution of the venting ports. To prevent damage during the ascent, it is necessary to know the maximum pressure jump produced by a fixed venting geometry, or, conversely, to size the venting holes for the maximum allowed pressure jump. Especially sensitive to pressure loading are multilayer insulations (MLIs) because they can deflect permanently or even tear. ${ }^{2}$ Thus, typical spacecraft qualification procedures include the determination of integrity against pressure loads by either calculations or tests.

A model for venting design, which is required on all spacecraft, also could help to reduce the vent sizes, thus helping in contamination control.

Previous published works are concerned with very detailed theoretical models that include nonhomogeneous distribution of the thermodynamic properties, internal flow, and the heat transfer mechanism from the walls into the closed domain., ${ }^{3,4}$ Their results are too complicated for engineering purposes in comparison to an explicit analytic expression. Simpicr models that retain the main physical phenomena are preferable. The high Knudsen number regime has also been modeled by other authors for particular geometric configurations, ${ }^{2}$ yielding analytic solutions that are of very restricted applicability when considering structural loads on general geometries.

The present article is a theoretical study of the discharge process, with special attention to the relevant parameters and design criteria for space systems. The small-pressure-jump case, which is the most desirable situation, allows for an asymptotic analysis, yielding simple analytic expressions of immediate engineering applicability. The validity range of this solution has been obtained and discussed in terms of the only relevant parameter: the ratio of the characteristic venting time to that related to the fairing pressure variation. The method developed was implemented in the UPM-Sat 1, a 
scientific and educational microsatellite ${ }^{5-7}$ developed by the Universidad Politécnica de Madrid, Spain, and launched on July 7, 1995, in the 75 th night of the Ariane 4.

\section{Analysis}

Consider a spacecraft or any equipment box under the launcher fairing as a reservoir discharging through a hole into an atmosphere of varying pressure defined by a given law $P_{e}(t)$, as shown in Fig. 1 . The following model gives us the necessary equations to describe the process.

The model presented is based mainly on mass conservation and polytropic evolution inside the container, with additional considerations of secondary relevance, such as exit conditions and empirical pressure-loss coefficients of the holes. The thermodynamic properties in the reservoir are considered homogeneous, and no spatial gradients are taken into account. This is a very idealized picture of the evolution in the container because heat transfer from the walls induces a convective flow, ${ }^{3}$ which is enhanced during the launch by the additive effect of the gravity field and the launcher acceleration. ${ }^{3,4}$ In the homogeneous model, no gradients are allowed in the container, but heat transfer with the spacecraft walls is modeled in an approximate way by the polytropic coefficient, which in the worst case is accepted as being close to the isothermal case. Modeling of the convection would increase the complexity greatly and obscure the discharge process itself. This is important whenever liquefaction has to be avoided or in active temperature-controlled systems, such as blow-down tunnels or life-support systems, which is not the case in this study. Furthermore, the temperature profile inside the fairing is not considered as it is not relevant to the reservoir discharge conditions because the fairing pressure is assumed to be known. However, the fairing temperature could change the spacecraft walls' temperature. For adiabatic behavior $(n=\gamma)$, there would be no influence in the spacecraft internal evolution, but in the case $n \neq \gamma$, the internal temperature will tend to follow the temperature of the container walls and, thus, the fairing temperature, especially in the isothermal limit when $n=1$. This fairing temperature variation has not been considered on the grounds that temperature data of most launchers show variations on the fairing walls of less than $10 \mathrm{~K}$ around the initial value during the first $100 \mathrm{~s}$ of the launch. ${ }^{8}$

Implicitly, continuum flow and perfect gas have been assumed, inasmuch as the analysis is not intended for the very low-pressure range, where the continuum evolves to a free-molecule flow. This range has not been considered because of its low impact when dealing with structural pressure loads. It can be necessary when outgassing modeling is required. Analytical and experimental results have been obtained for this regime by other authors. ${ }^{2}$

\section{Model of the Reservoir}

For a homogeneous distribution of the thermodynamic properties, the mass variation in the reservoir is

$$
V \frac{\mathrm{d} \rho_{01}}{\mathrm{~d} t}=-\rho_{h} S U_{h}
$$

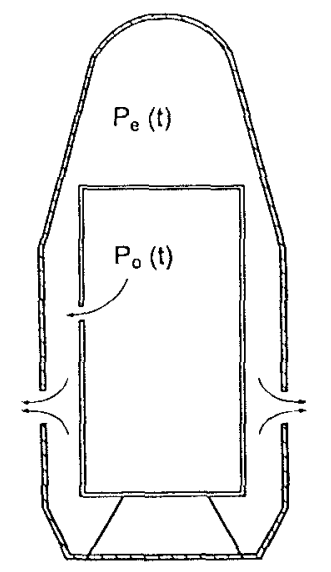

Fig. 1 Model of a spacecraft or equipment box with inner pressure $P_{0}(t)$ discharging inside the launcher fairing at pressure $P_{e}(t)$ during the launch into orbit.
Under appropriate conditions, the evolution of the thermodynamic properties within the reservoir can be described by a polytropic law:

$$
\frac{P_{0}(t)}{\rho_{0}(t)^{n}}=\frac{P_{i}}{\rho_{i}^{\prime \prime}}
$$

where $n$ varies from 1 (for isothermal cvolution) to $\gamma$ (for adiabatic evolution) and the subscripts $i$ and 0 stand for initial and instantaneous conditions in the reservoir, respectively.

\section{Flow Through the Hole: Compressible Model}

The flow through the hole can be considered quasisteady because the residence time of the flow particles in the hole is supposed to be much smaller than the characteristic variation time of the boundary conditions. For a first physical description, this flow can be taken as one dimensional, adiabatic, inviscid, and laminar, up to the exit section, that is, not including the jet. This allows us to write the isentropic relations

$$
\left(a_{0} / a_{h}\right)^{2}=\left(P_{0} / P_{h}\right)^{(\gamma-1) / \gamma}=\left(\rho_{0} / \rho_{h}\right)^{\gamma-1}=1+[(\gamma-1) / 2] M_{h}^{2}
$$

where $M_{h}$ is the Mach number at the exit section, defined as

$$
M_{h}=\frac{U_{h}}{\sqrt{\gamma P_{h} / \rho_{h}}}
$$

Equations (3) and (4) allow us to evaluate $\rho_{h}$ and $U_{h}$ at the exit section. The venting hole can be considered as a converging nozzle, which will be blocked at its maximum flow rate if critical conditions are reached at the output section $\left(M_{h}=1\right)$. Nozzle block will occur for a determined value of the outer pressure $P_{\varepsilon}=P_{b}$, related to the inner pressure $P_{0}$, which can be easily deduced from Eq. (3):

$$
\left(P_{b} / P_{0}\right)=[2 /(\gamma+1)]^{\gamma /(\gamma-1)}
$$

For $\gamma=1.4$, this is $P_{b} / P_{0}=0.5283$. In the case of $P_{e}>P_{b}$, the flow at the hole exit is subsonic, it is not choked, and the pressure at the output $P$ equals the outer pressure $P_{e}$. Introducing Eqs. (2-4) into Eq. (1) with the condition $P_{h}=P_{e}$ yields the equation describing the evolution of the density in the reservoir:

$$
\begin{aligned}
\frac{\mathrm{d} \rho_{0}}{\mathrm{~d} t} & =-\frac{S}{V}\left(\frac{2 \gamma}{\gamma-1} \frac{P_{i}}{\rho_{i}^{n}}\right)^{\frac{1}{2}} \rho_{0}^{(n+1) / 2}\left[\frac{P_{i}}{P_{c}}\left(\frac{\rho_{0}}{\rho_{i}}\right)^{n}\right]^{-(\gamma+1) / 2 \gamma} \\
& \times\left\{\left[\frac{P_{i}}{P_{r}}\left(\frac{\rho_{0}}{\rho_{i}}\right)^{n}\right]^{(\gamma-1) / \gamma}-1\right\}^{\frac{1}{2}}
\end{aligned}
$$

whereas in the case of choked flow, the output is sonic, $M_{h}=1$, and an alternative expression is obtained for the density:

$$
\frac{\mathrm{d} \rho_{0}}{\mathrm{~d} t}=-\frac{S}{V}\left[\left(\frac{2}{\gamma+1}\right)^{(\gamma+1) /(\gamma-1)} \frac{\gamma P_{i}}{\rho_{i}^{n}}\right]^{\frac{1}{2}} \rho_{0}^{(n+1) / 2}
$$

This equation is directly integrable separating variables. Equations (6) and (7) are mutually exclusive, depending on whether the pressure ratio $P_{r}(t) / P_{0}(t)$ corresponds to choked flow. The discharge can become either choked or unchoked during the process: thus, a conditional integration must be performed. The flow starts with unchoked conditions because at the beginning the pressure jump is zero. The flow choking occurs when the pressure under the fairing, which is monotonously decreasing, reaches the value $P_{b}=0.528 P_{0}$. At the final states, when both pressures are very small, the pressure ratio $P_{e} / P_{0}$ becomes larger than 0.528 and again the flow becomes unchoked. This last stage has not been considered in this study because both pressures and the absolute pressure jump are negligible from the point of view of wall loading.

Both Eqs. (6) and (7) are nonlinear, containing the parameters of the problem, and Eq. (6) includes the outer pressure $P_{t}(t)$ as a forcing term. 


\section{Flow Through the Hole: Incompressible Model}

Choking of the discharge is related to a high-pressure jump, so that in practice this situation must be avoided and, in fact, a smallpressure jump is preferred to decrease structural loads. In the following, this more practical case of a small-pressure jump is considered. The real incompressible (low-speed) flow conditions through the hole are introduced by replacing Eqs. (3) and (4) by $\rho_{0}=\rho_{h}$ and $U_{h}$ from

$$
\Delta P=P_{0}-P_{e}=\frac{1}{2} \rho_{10} U_{h}^{2} \xi
$$

where $\xi$ is the total pressure loss coefficient, which can be evaluated as $\xi \approx 1.5$ for a standard sharp-edged hole or $\xi \approx 1.0$ for a wellrounded hole. ${ }^{9.10}$ The density of the gas reaching the hole can be approximated by the reservoir density $\rho_{0}$ in the small-pressure-jump case. This incompressibility hypothesis will be discussed and evalwated in the next sections. Introducing Eq. (8) into Eq. (1) produces the evolution equation

$$
\frac{\mathrm{d} \rho_{0}}{\mathrm{~d} t}=-\frac{S}{V} \sqrt{\frac{2 \rho_{0} \Delta P}{\xi}}
$$

which replaces Eqs. (6) and (7). Further considerations concerning $\Delta P$ are required for solving Eq. (9) in terms of $\rho_{0}$.

\section{Nondimensional Form of the Equations}

The problem can be rewritten by using variables made dimensionless with the characteristic venting time $t_{c}$, the density $\rho_{i}$, and the pressure $P_{i}$ as follows:

$$
\begin{gathered}
t=t_{c} T, \quad \rho_{0}=\rho \rho_{i}, \quad P_{0}=p P_{i} \\
P_{k}=p_{e} P_{i}, \quad \Delta P=\delta P_{i}
\end{gathered}
$$

Then Eq. (9) leads to

$$
\frac{\mathrm{d} \rho}{\mathrm{d} T}=-t_{c} a_{i} \frac{S}{V} \sqrt{\frac{2 \rho \delta}{\gamma}}
$$

where $a_{i}=\sqrt{ }\left(\gamma P_{i} / \rho_{i}\right)$ is the speed of sound in the reservoir at the initial conditions. Defining the characteristic venting time $t_{c}$ as

$$
t_{c}=\left(V / S a_{i}\right) \sqrt{\xi \gamma / 2}
$$

Eq. (11) can be rewritten to give

$$
\frac{\mathrm{d} \rho}{\mathrm{d} T}=-\sqrt{\rho \delta}
$$

which has to be solved with the initial condition $\rho(0)=1$. In the same manner, the nondimensional form of Eqs. (6) and (7) is

$$
\begin{aligned}
P_{e} & >P_{b} \Rightarrow \frac{\mathrm{d} \rho}{\mathrm{d} T}=-\left(\frac{\gamma}{\gamma-1} \rho^{n+1}\right)^{\frac{1}{2}}\left(\frac{\rho^{n}}{p_{e}}\right)^{-(\gamma+1) / 2 \gamma} \\
& \times\left[\left(\frac{\rho^{n}}{p_{e}}\right)^{(\gamma-1) / \gamma}-1\right]^{\frac{1}{2}} \\
P_{e} & \leq P_{b} \Rightarrow \rho=-\{[2 T /(n+3)] \\
& \left.\times \sqrt{(\gamma / 2)[2 /(\gamma+1)]^{(\gamma+1) /(\gamma-1)}}\right\}^{-2 /(n+3)}+C\left(T_{0}\right)
\end{aligned}
$$

where $C\left(T_{0}\right)$ is an integration constant. Here the characteristic time is

$$
t_{c}=\left(V / S a_{i}\right) \sqrt{\gamma / 2}
$$

which is slightly different from that defined in Eq. (12). However, considering that the total pressure loss coefficient $\xi$ is close to one and, furthermore, that it appears in Eq. (12) under the square root, its influence can be neglected or included in the effective hole section $S$. In the following, $\xi=1$ has therefore been taken, allowing for a unified parametric description of both the incompressible and compressible discharge regimes.

\section{Asymptotic Solution for Small $\delta$}

As the pressure jump should be small ( $P_{0}$ close to $\left.P_{e}\right)$ in Eq. (13), the assumption $\delta \ll 1$ allows us to obtain an asymptotic solution of the problem. Therefore, the variables of the problem can be expressed as a series expansion in powers of a small parameter $v \ll 1$ as follows:

$$
\begin{gathered}
p=p_{e}+v p_{1}+\mathcal{O}\left(v^{2}\right) \\
\delta=p-p_{e}=\nu p_{1}+\mathcal{O}\left(v^{2}\right) \\
\rho=p^{1 / n}=p_{e}^{1 / n}\left[1+(\nu / n)\left(p_{1} / p_{e}\right)+\mathcal{O}\left(v^{2}\right)\right]
\end{gathered}
$$

An essential assumption is that the pressure variation in the fairing $P_{t}$ contains in its argument its own characteristic time $t_{p}$; that is, the argument should be of the type $t / t_{p}$ or $K T$, where $K=t_{c} / t_{p}$ is the ratio of characteristic times. Therefore, using Eqs. (16) in Eq. (13) leads to

$$
\begin{aligned}
\frac{\mathrm{d} \rho}{\mathrm{d} T} & =\frac{1}{n} p_{e}^{-1+1 / n} \dot{p}_{e} K+\mathcal{O}(K v)=-\sqrt{\rho \delta} \\
& =-\left\{v p_{1} p_{e}^{1 / n}\left[1+\frac{v}{n} \frac{p_{1}}{p_{e}}+\mathcal{O}\left(v^{2}\right)\right]\right\}^{\frac{1}{2}}
\end{aligned}
$$

where the dot represents derivation with respect to the argument $K T$. To match the expansions on both sides of Eq. (17), it is required that $v=K^{2} \ll 1$. With this in mind and identifying terms on both sides of Eq. (17), we reach an equation that describes the small-pressurejump discharge process for a general external evolution verifying $K^{2} \ll \mathrm{I}$ :

$$
p_{1}=\left(1 / n^{2}\right) p_{e}^{-2+1 / n} \dot{p}_{e}^{2}
$$

Assuming $n=1$, which will be discussed in the next sections, Eq. (18) simplifies to

$$
p_{1}=\dot{p}_{e}^{2} / p_{e}
$$

In the limit of the small-pressure jump $(\delta \ll 1)$, it can be shown that the compressible nonchoked formulation equation (14) coincides with the incompressible model equation (13) when $\xi=1$, and, therefore, the results obtained in this section are valid for both approaches. This coincidence will be observed again in the next section by comparing numerical results obtained from the compressible formulation with the asymptotic equation (18).

\section{Fairing Pressure Profiles: Case of the UPM-Sat 1}

The pressure profiles under the fairing depend mainly on the evolution of the launch vehicle through the atmosphere and the geometry of the venting holes. From a rigorous point of view, both discharge processes, the discharge of the fairing into the atmosphere and the evacuation of the air trapped in the payloads and equipment boxes, should be considered simultaneously as coupled processes with their own characteristic times. The analysis could then be performed with a simple model of series and parallel discharging reservoirs. Instead of a single equation for the conservation of mass, a system consisting of as many equations as reservoirs could be written. The equations for the intermediate reservoirs, as the fairing, are the only ones that would contain coupled terms due to the inflowing air coming from the payloads.

Depending on the accuracy level of the calculations, the mutual influence of the external flow around the fairing during the ascent trajectory and the discharging jets may be modeled. For engineering purposes the knowledge of the static pressure profile on the fairing outer surface would be enough, inasmuch as this would serve as a first approximation to the driving mechanism of the discharge of the fairing.

However, the air mass trapped in the spacecraft is usually included in the total air mass under the fairing, which allows us to decouple the processes. In practice, the pressure profiles under the fairing are prescribed for all launchers independently of the spacecraft to be launched. To establish a general pressure profile under the fairing, pressure data of some launchers have been compiled. The result 


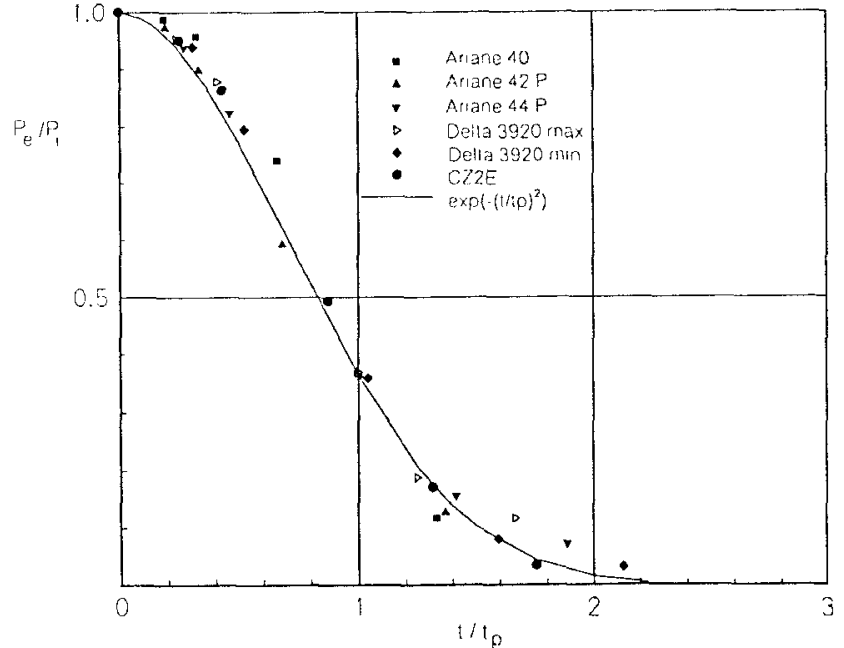

Fig. 2 Pressure profiles $P_{e}(t)$ under the fairing, made dimensionless with the initial pressure $P_{i}$ and scaled with the characteristic time $t_{p}$ of each launcher.

is shown in Fig. 2, where all series of the points are scaled with the initial pressure $P_{i}$ and the profile characteristic time $t_{p}$ of each launcher. $^{8}$

These pressure profiles can be represented in an approximate way by the following expression:

$$
p_{e}=e^{-(K T)^{2}}
$$

This simple law fulfills the following four conditions in regard to the matching with pressure profile launcher data: 1) equal value of the function $\left(p_{e}=1\right)$ and 2$)$ the time derivative $\left(\mathrm{d} p_{e} / \mathrm{d} t=0\right)$ at the initial time, 3) equal value at the reference point $t=t_{p}, p_{e}(K T=$ $1)=e^{-1}$, and 4) zero value for its asymptotic derivative $(t \rightarrow \infty)$. The characteristic time for the fairing pressure variation $t_{p}$ is determined from flight data using condition 3 . Although no statistical relevance can be given to the data in Fig. 2, the launcher profiles, selected on the basis of a rather high dispersion in their respective pressure characteristic times, show very good agreement with the exponential law.

The values of $t_{p}$ for the launchers considered in Fig. 2 are as follows: 1) Ariane 40 (sun-synchronous orbit), $t_{p}=75 \mathrm{~s} ; 2$ ) Ariane $42 \mathrm{P}, t_{p}=73 \mathrm{~s}$; 3) Ariane $44 \mathrm{P}, t_{p}=53 \mathrm{~s}$; 4) Delta 3920 maximum, $t_{p}=57 \mathrm{~s}$; 5) Delta 3920 minimum, $t_{p}=44 \mathrm{~s}$; and 6) CZ2E $t_{p}=$ $58 \mathrm{~s}$.

From Eqs. (16b) and (18), keeping in mind that the dots represent derivation with respect to the argument $K T$, and using Eq. (20), $p_{1}$ and $\delta_{a}$ are obtained as

$$
\begin{gathered}
p_{1}=[(2 / n) K T]^{2} e^{-(K T)^{2} / n} \\
\delta_{a}=K^{2} p_{1}=K^{2}[(2 / n) K T]^{2} e^{-(K T)^{2} / n}
\end{gathered}
$$

The maximum value of the asymptotic pressure jump $\delta_{a}=\delta_{\text {max }}$ appears at $T=T_{\max }$, where $T_{\max }$ is the solution of $\mathrm{d} \delta_{a} / \mathrm{d} T=0$. Derivation in Eq. (21) leads to

$$
T_{\max }=\sqrt{n} / K
$$

and, hence, from Eq. (21b)

$$
\delta_{\max }=4 K^{2} / n e
$$

A useful conclusion is that $T_{\max } K=t_{\max } / t_{n}=\sqrt{ } n$ and, therefore, the maximum pressure jump predicted by the asymptotic solution occurs at $t=t_{\max } \approx t_{p}$. The value of $n$ is 1.4 for adiabatic and 1 for isothermal processes. The worst case (larger pressure jump) is $n=1$, as can be seen in Eq. (22b).

The characteristic venting time for the UPM-Sat 1 (Table 1) is $t_{c}=1.4 \mathrm{~s}$. In the case of Ariane 40 (sun-synchronous launch), at $t=t_{p}=75 \mathrm{~s}, P_{t}\left(t_{p}\right)=P_{i} e^{-1}=368$ mbar. Consequently, $K=$ $1.4 / 75 \approx 1.9 \times 10^{-2} \ll 1$. For this value of $K$ and assuming $n=1$,
Table 1 UPM-Sat 1 venting data

\begin{tabular}{lccr}
\hline$S$ & $V$ & $a_{i}$ & $\xi$ \\
\hline $24 \times 10^{-5} \mathrm{~m}^{2}$ & $0.13 \mathrm{~m}^{3}$ & $335 \mathrm{~m} / \mathrm{s}(\gamma=1.4)$ & 1 \\
\hline
\end{tabular}

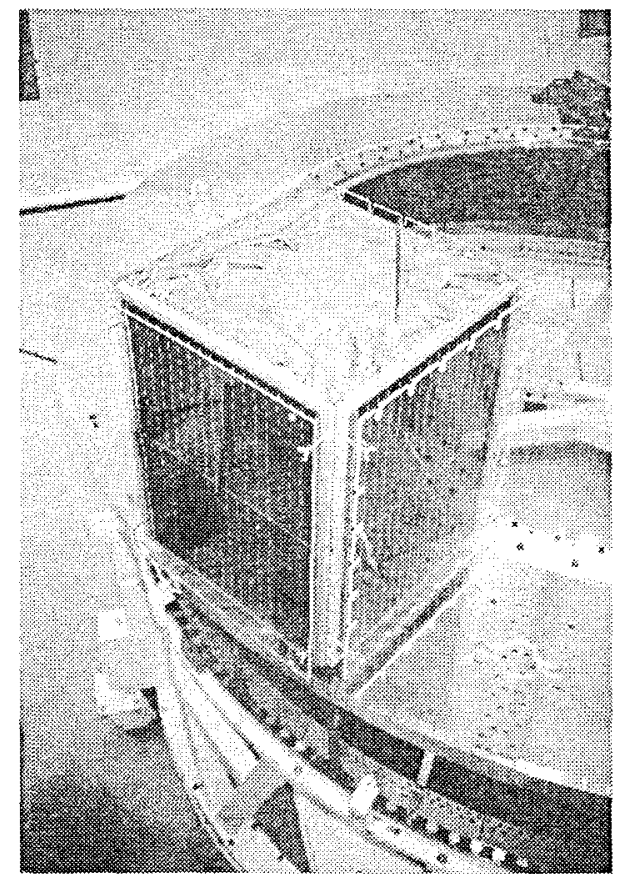

Fig. 3 UPM-Sat 1 mounted for launch on the ASAP platform of the 75 th Ariane 4 flight.

we get $\delta_{\max }=5.4 \times 10^{-4}$, which represents $\Delta P_{\max }=54 \mathrm{~Pa}$, a small load for the closing structural panels of the satellite. In Fig. 3, a general view of the UPM-Sat 1 mounted on the Ariane Structure for Auxiliary Payloads (ASAP) platform of Ariane 4 is shown, being remarkable for the closed configuration, typical for microsatellites.

\section{Asymptotic Solution Validity Range: Choking Effect}

and Numerical Results Comparison

Numerical integration of Eq. (14), conditionally coupled with Eq. (15), can be used to determine the range of the parameter $K$ for which the asymptotic solution equation (18) is valid. A standard Runge-Kutta method has been applied with $K$ and $n$ as the relevant parameters. The Ariane pressure profile has been used by means of the analytic approximation equation (20), and the ratio of specific heats $\gamma=1.4$ has been assumed for air.

In Figs. $4 a$ and $4 b$, numerical and asymptotic results are compared for different values of the ratio of characteristic times $K$, for the worst case $n=1$. In the horizontal axis, the stretched time $K T$ is used for proper scaling. Note that $K T=t / t_{p}$. Both dimensionless inner and fairing pressures start from $p=p_{r}=1$. The asymptotic solution for the pressure jump $\delta_{a}$, as well as the numerical solution $\delta_{n}$, is scaled with the maximum value of the asymptotic solution $\delta_{\max }$, given by Eq. (22b). Good agreement between both solutions is observed for $K<0.1$, especially concerning the maxima of the pressure jump and its location at $K T=1$. In fact, for $K=0.05$ (see Fig. 4a), $\delta_{a}$ and $\delta_{n}$ are practically coincident. For small $K$ the discharge characteristic time $t_{c}$ is negligible compared to $t_{p}$, so that the reservoir remains in quasiequilibrium with the fairing pressure and the outflow speed is low. As a consequence of the results, the incompressibility hypothesis can be accepted as correct for this range of $K$, as already stated in preceding sections. Moreover, no choking can be observed until the pressure $p_{e}$ has decreased to extremely low levels, where the pressure loads are negligible and of no structural relevance. In particular for $K=0.1$, choking $\left(p / p_{e}=1.89\right)$ appears at $K T=2.92$ and $p_{e}=2 \times 10^{-4}\left(P_{e}=20 \mathrm{~Pa}\right)$. From the choking point on, the inner pressure $p$ follows the $T^{-1 / 2}$ law described by Eq. (15) when $n=1$. Observe that, in the range described so far, the dimensionless pressure jump $\delta_{a}$ varies as $K^{2}$. 


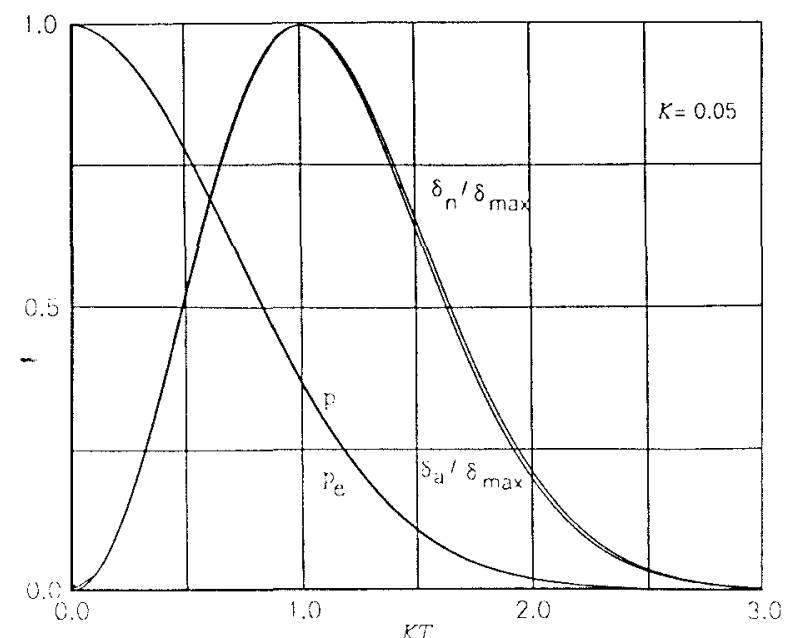

a) $\delta_{\max }=3.7 \times 10^{-3}$

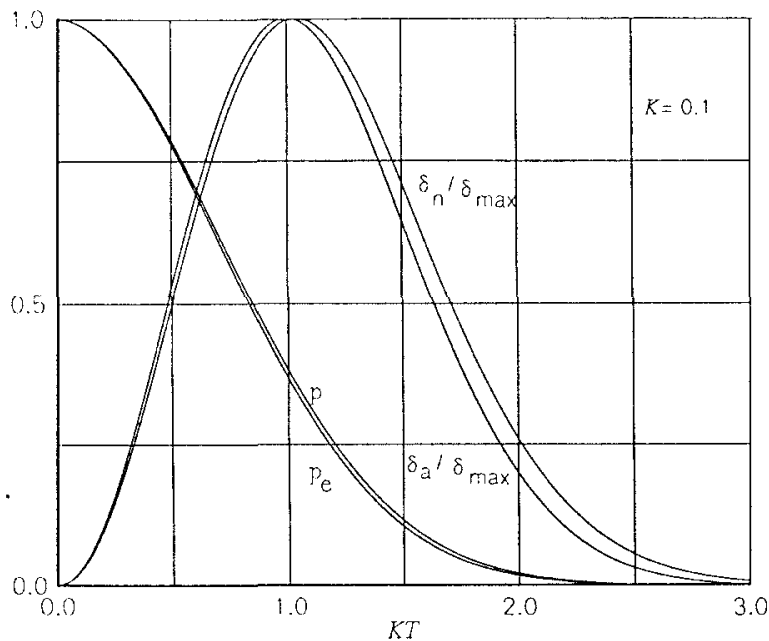

b) $\delta_{\max }=1.5 \times 10^{-2}$

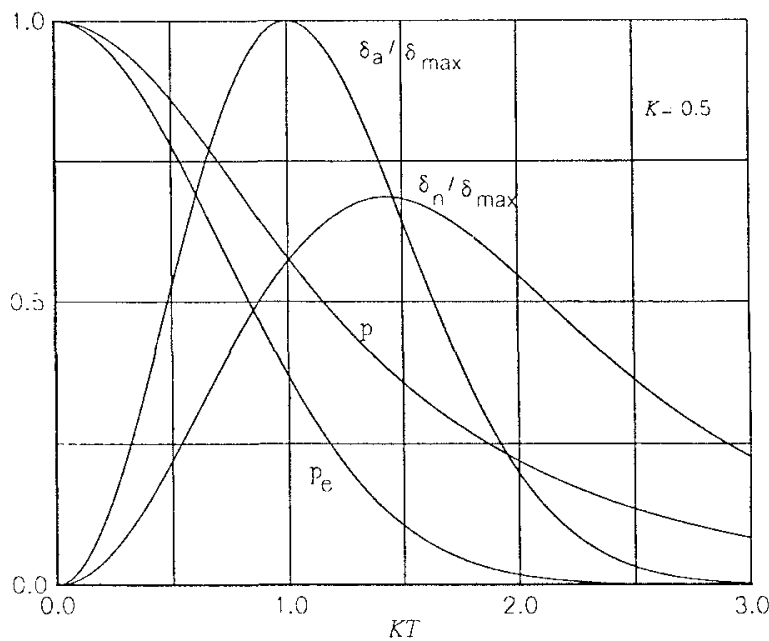

c) $\delta_{\max }=0.37$

Fig. 4 Variation with the dimensionless stretched time $K T$ of the dimensionless variables: inner pressure $p$, fairing pressure $p_{\ell}$, numerical solution $\delta_{n}$, and asymptotic solution $\delta_{a}$ for the pressure jump; $n=1$.

In Fig. $4 c(K=0.5)$, however, the effect of compressibility, and particularly choking, is quite noticeable. As the pressure jump becomes of the same order as the pressure itself, the condition $\delta \ll 1$ does not hold and the asymptotic solution is no longer valid. The computed solution shows a pressure jump maximum smaller than the one predicted by the asymptotic solution, and it occurs at higher times, thus giving longer venting times. The choking point moves to lower times for increasing $K$. Obviously, because

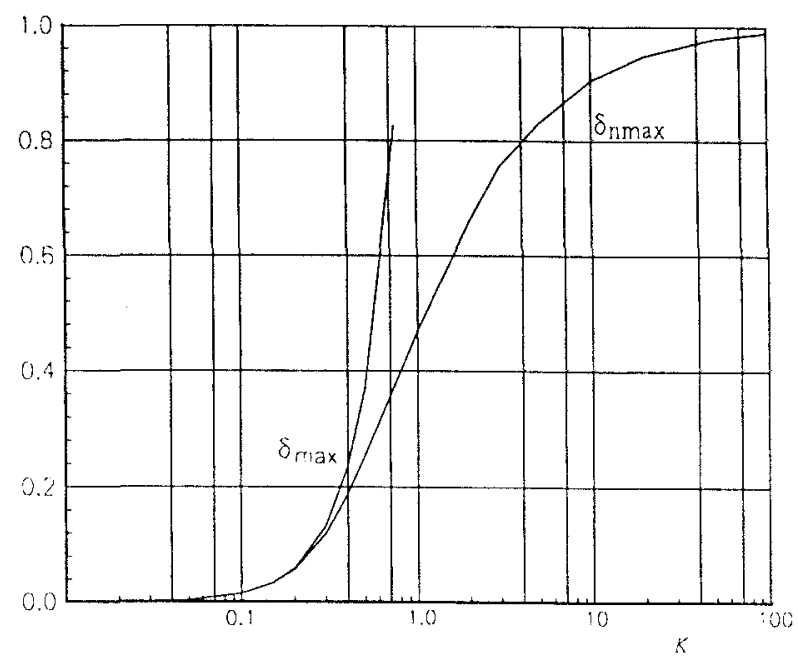

Fig. 5 Maximum dimensionless pressure jump obtained from numerical results $\delta_{n \max }$ and from the asymptotic solution $\delta_{\max }$ as a function of the ratio of characteristic times $K$.

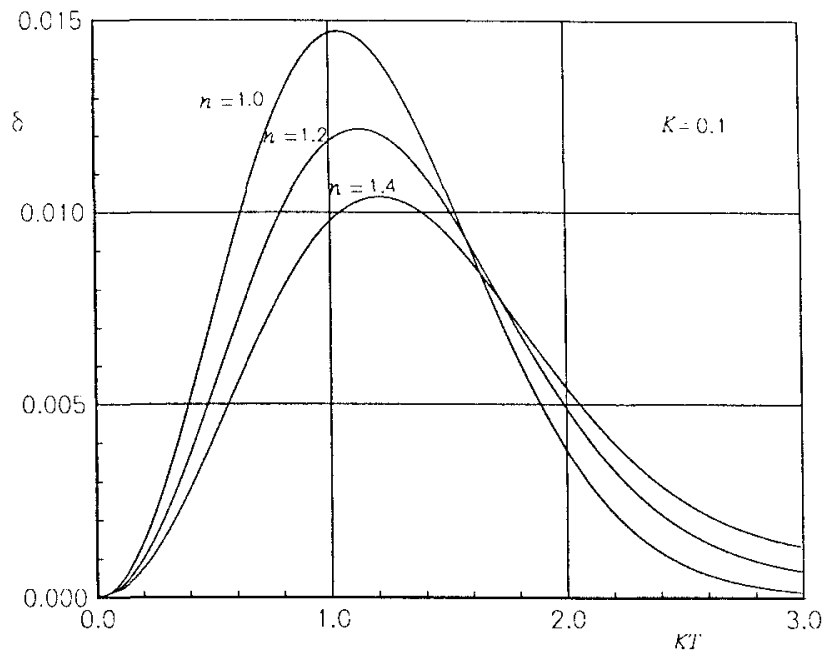

Fig. 6 Effect of the polytropic coefficient $n$ on the variation of the dimensionless pressure jump $\delta$ with stretched time $K T$ for $K=0.1$.

$K=t_{c} / t_{p} \propto V\left(S a_{i} t_{p}\right)^{-1}$, for bigger container volumes, smaller orifices, or quicker fairing depressurization, the pressure jump will rise higher and choking will appear sooner. For $K=0.5$, the choking point is located at $K T=1.16$, even before the occurrence of the maximum pressure difference.

The difference between the asymptotic $\delta_{\max }$ and the numerical $\delta_{n \max }$ solutions is more evident in Fig. 5, where they are plotted against the ratio of characteristic times. When $K$ is such that choking advances into the region where the pressure jump is still close to its maximum, e.g., $K T \approx 2$, then the slope of the numerical result $\delta_{n \max }$ in Fig. 5 peaks sharply, starting the divergence between the asymptotic and numerical results. The numerical solution is essentially correct in the non-small-pressure-jump range because it retains compressibility effects in the model. The validity range of the asymptotic approximation equation (18) is, thus, $K<0.1$, and this limit is determined by the advance of the choking point into the time interval where fairing pressure is not negligible $(K T<2)$. Observe that, if both characteristic times are the same, that is, $K=1.0$, the maximum pressure jump is approximately $0.5 P_{i} \approx 5 \times 10^{4} \mathrm{~Pa}$, which can result in a quite heavy pressure loading on the container walls. In the case of an MLI, this load could even tear the insulation layers. ${ }^{2}$ As can be drawn from the preceding discussion, the driving parameter is $K$, the ratio of characteristic times.

Concerning the polytropic coefficient $n$ of the evolution inside the container, it plays a limited role, at least in the small-pressure-jump range, as can be deduced from Fig. 6, where numerical results are plotted for different values of $n$. The most critical case is $n=1$, the 
isothermal case, as expected. A physical explanation for this effect has to be attributed to the beneficial temperature decrease in an isentropic discharge. ${ }^{3,11}$ As can be observed in Fig. 6, the maximum pressure difference $\delta$ decreases as $n^{-1}$ and delays as $n^{1 / 2}$ [see Eq. (22)].

Concerning the external pressure profile, the asymptotic solution is valid for any fairing pressure profile $P_{t}(t)$ and reservoir venting features whose characteristic variation times $t_{p}$ and $t_{c}$ satisfy $K<$ 0.1 . Equations (18) and (16) give the general asymptotic solution, whereas Eq. (21) and the numerical results shown correspond to the Ariane 40 launch profile $\left(t_{p}=75 \mathrm{~s}\right)$.

\section{Conclusions}

The air discharge process of a spacecraft or any equipment box during launch must be observed from the structural point of view. If the evacuation is not fast enough, excessive pressure loading can result in damage of elements exposed to the pressure jump. This manifests in closed-type spacecraft structures, as in the case of the UPM-Sat 1 . Engineering criteria are then necessary to evaluate venting rates or pressure loads during the launch.

A model of the discharge process of a reservoir into a decreasing time-dependent pressure atmosphere has been proposed. The case of small-pressure-jump $\delta \ll 1$, which is the relevant case for spacecraft design, has led to a simple asymptotic solution that relies on a single nondimensional parameter $K$, the ratio of the characteristic times of the reservoir venting to fairing pressure variation. This asymptotic solution, which in this limit behaves as $\delta \sim K^{2}$, serves to evaluate pressure loads for a given geometry or to size the venting holes for a given maximum pressure jump. All other parameters studied, such as the pressure loss coefficient or the polytropic coefficient, play a secondary role.

The asymptotic solution has also been proven to be in accordance with incompressible flow conditions, inasmuch as the speed through the hole is small enough. Compressibility of the flow through the holes and especially choking become relevant for $K>0.1$, producing a sudden increase in the pressure jump maximum. However, the case $K>0.1$, although interesting in regard to pressurizedtank discharge, has not been pursued further inasmuch as the focus is on spacecraft venting loads.

Numerical integration of the compressible model equations, including the effect of choking, has been used to obtain the range of validity of the asymptotic solution. The limit of this range, $K \approx 0.1$, is determined by the advance of the choking point into the time interval where the pressure jump is still close to its maximum.

The analysis performed does not depend on the particular pressure profile, and a closed-form solution that is applicable to any pressure profile under the fairing has been obtained; that is, it is valid for any launcher with only the restriction that $K=t_{c} / t_{p}<0.1$. In addition, a general behavior of the pressure profile of some launchers, which is described by an exponential law, has been found. This result helps to reduce the number of parameters needed for analysis or design.

This similar fairing pressure profile and the asymptotic solution for the pressure jump are the design tools that serve for sizing the discharge holes or estimating depressurization loads. 